\title{
Genetic loci for grain protein and gluten content in Russian spring wheat varieties
}

\author{
Leonova I.N. ${ }^{1 *}$, Kiseleva A.A. ${ }^{1,2}$, Stasyuk A.I. ${ }^{1,2}$, Salina E.A. ${ }^{1,2}$ \\ ${ }^{1}$ Institute of Cytology and Genetics, SB RAS, Novosibirsk, Russia \\ ${ }^{2}$ Kurchatov Genomic Center of the Institute of Cytology and Genetics, SB RAS, Novosibirsk, Russia \\ *email: leonova@bionet.nsc.ru
}

The nutritional value of wheat and the quality of flour are largely dependent on the grain protein content (GPC). The development of high-quality wheat varieties, identification of new genetic loci for GPC remains a main task for modern wheat breeding. The aim of this study was analysis of composition of Russian spring bread wheat varieties on genetic loci controlling GPC and gluten content. A panel of wheat varieties was phenotyped at the two localities of Western Siberian region during three years. The GPC of the varieties varied from 12.9 to $17.5 \%$ while gluten content changed from 21.3 to $39.3 \%$ of the total protein content. Heritability of the GPC and gluten was $\sim 0.62$, confirming that environment strongly influence the traits, however the genetic component was also high. Wheat varieties were characterized by markers specific for alleles of loci encoding high molecular weight glutenins (Glu-A1, Glu-B1, and Glu-D1). Summarizing the results of the marker analysis, it was concluded that 27 of 92 varieties contain combinations of Ax2, Dx5 and Dy10 subunits positively effect on baking qualities. Genome-wide association study performed by MLM model revealed SNP markers in 6A, 7B, and 4A chromosomes significantly associated with both protein and gluten content. SNPs, mapped at 6AS chromosome may be linked with NAM-A1 (GPC1) loci - a key factor determining protein and gluten content in common wheat. The obtained results also suggest the presence of new genetic factors that affect the GPC and gluten content in the genome of the bread wheat varieties.

Acknowledgements: This study was supported by the Russian Scientific Foundation (Project No. 21-76-30003) and budget project of the Institute of Cytology and Genetics, SB RAS No. 0259-2021-0012. 